\title{
Nonlocal electrodynamics of rotating systems
}

\author{
Bahram Mashhoon \\ Department of Physics and Astronomy, \\ University of Missouri-Columbia, \\ Columbia, Missouri 65211, USA
}

(Received 25 March 2005; published 8 November 2005)

\begin{abstract}
The nonlocal electrodynamics of uniformly rotating systems is presented and its predictions are discussed. In this case, due to paucity of adequate experimental data, the nonlocal theory cannot be directly confronted with observation at present. The approach adopted here is therefore based on the correspondence principle: the nonrelativistic quantum physics of electrons in circular "orbits" is studied. The helicity dependence of the photoeffect from the circular states of atomic hydrogen is explored as well as the resonant absorption of a photon by an electron in a circular "orbit" about a uniform magnetic field. Qualitative agreement of the predictions of the classical nonlocal electrodynamics with quantum-mechanical results is demonstrated in the correspondence regime.
\end{abstract}

DOI: 10.1103/PhysRevA.72.052105

PACS number(s): 03.30.+p, 11.10.Lm, 03.65.Sq, 04.20.Cv

\section{INTRODUCTION}

Imagine a background inertial reference frame with coordinates $x^{\mu}=(t, \mathbf{x})$ in Minkowski spacetime. An accelerated observer follows a worldline $x^{\mu}(\tau)$, where $\tau$ is the proper time along its path. According to the standard theory of relativity, the accelerated observer-at each instant along its worldline-is equivalent to an otherwise identical momentarily comoving inertial observer $[1,2]$. This hypothesis of locality implies that an accelerated observer passes through a continuous infinity of hypothetical momentarily comoving inertial observers. Lorentz invariance can then be locally extended to noninertial observers via this basic assumption. Each inertial observer is endowed with an orthonormal tetrad frame; therefore, the hypothesis of locality implies that an accelerated observer carries an orthonormal tetrad frame $\lambda_{(\alpha)}^{\mu}(\tau)$ along its worldline such that $\lambda^{\mu}{ }_{(0)}=d x^{\mu} / d \tau$ is its temporal axis and $\lambda^{\mu}{ }_{(i)}, i=1,2,3$, are the unit axes of its local spatial frame.

The motion of the tetrad frame along the worldline of the accelerated observer may be expressed as

$$
\frac{d \lambda^{\mu}(\alpha)}{d \tau}=\Phi_{\alpha}^{\beta} \lambda^{\mu}{ }_{(\beta)},
$$

where $\Phi_{\alpha \beta}(\tau)$ is the antisymmetric acceleration tensor. In analogy with the Faraday tensor, $\Phi_{\alpha \beta} \rightarrow(-\widetilde{\mathbf{g}}, \widetilde{\mathbf{\Omega}})$; that is, the "electric" part $\left(\Phi_{0 i}=\widetilde{g}_{i}\right)$ consists of the translational acceleration of the observer, while its "magnetic" part $\left(\Phi_{i j}=\epsilon_{i j k} \widetilde{\Omega}^{k}\right)$ consists of the rotational frequency of the spatial frame with respect to a nonrotating (i.e., Fermi-Walker transported) tetrad frame. The scalar invariants $\widetilde{\mathbf{g}}(\tau)$ and $\widetilde{\mathbf{\Omega}}(\tau)$ characterize the rate of change of the state of the observer and may be used to construct the acceleration scales-i.e., acceleration length $\mathcal{L}$ and acceleration time $\mathcal{L} / c$ - of the observer. For an Earth-based laboratory, for instance, $\mathcal{L}=c^{2} / g_{\oplus} \cong 1$ light year is the translational acceleration length, while $\mathcal{L}=c / \Omega_{\oplus}$ $\cong 28 \mathrm{~A} . \mathrm{U}$. is the rotational acceleration length.

If all physical phenomena could be reduced to pointlike coincidences, then the hypothesis of locality would be strictly valid. Indeed, the hypothesis of locality originates from Newtonian mechanics, where the state of a point particle is characterized by its position and velocity. The accelerated particle and the momentarily comoving inertial particle have the same state; therefore, they are pointwise physically equivalent. Thus no new physical hypothesis is needed in the Newtonian treatment of accelerated systems. However, for wave phenomena we expect deviations from the hypothesis of locality that would be proportional to $\lambda / \mathcal{L}$, where $\lambda$ is the wavelength of the radiation. To illustrate this viewpoint, consider the measurement of the frequency of incident radiation by an accelerated observer. At least a few oscillations of the incident wave must be received by the observer before a reasonable determination of its frequency can be made; however, during this time interval of $\sim \lambda / c$ the state of the observer has changed. Nevertheless, this may be ignored if $\lambda$ is sufficiently small compared to $\mathcal{L}$. The consistency of this approach can be illustrated in the case of a classical particle of mass $m$ and charge $q$ that is accelerated by an external force $\mathbf{f}$. The accelerated charge radiates electromagnetic radiation of wavelength $\lambda \sim \mathcal{L}$, where $\mathcal{L}$ is the particle's acceleration length. It is expected that the hypothesis of locality is violated in the interaction of the particle with the electromagnetic field as $\lambda / \mathcal{L} \sim 1$. Thus the state of the particle cannot be characterized by its position and velocity as demanded by the hypothesis of locality. This is in agreement with the equation of motion of the particle, which in the nonrelativistic approximation may be expressed as

$$
m \frac{d \mathbf{v}}{d t}-\frac{2}{3} \frac{q^{2}}{c^{3}} \frac{d^{2} \mathbf{v}}{d t^{2}}+\cdots=\mathbf{f} .
$$

The dependence of this Abraham-Lorentz equation on the temporal derivative of the acceleration implies that the state of a radiating particle cannot be adequately characterized by its position and velocity.

It follows from these arguments that it is necessary to contemplate a generalization of the hypothesis of locality 
that would be adequate for all wave phenomena. To proceed, we restrict our attention to the measurement of an electromagnetic radiation field $F_{\mu \nu}$ by an accelerated observer, though the general approach is applicable to any radiation field [3]. According to the hypothesis of locality, the accelerated observer may be replaced at each instant by the momentarily comoving inertial observer for which the measured field is equivalent to the projection of $F_{\mu \nu}$ onto its tetrad frame. Consider the class of fields $F_{(\alpha)(\beta)}(\tau)$ measured pointwise by the hypothetical momentarily comoving inertial observers; then

$$
F_{(\alpha)(\beta)}(\tau)=F_{\mu \nu} \lambda^{\mu}{ }_{(\alpha)} \lambda^{\nu}{ }_{(\beta)} .
$$

Let $\mathcal{F}_{(\alpha)(\beta)}(\tau)$ be the electromagnetic field that is actually measured by the accelerated observer. The hypothesis of locality states that at each instant $\tau, \mathcal{F}_{(\alpha)(\beta)}(\tau)$ and $F_{(\alpha)(\beta)}(\tau)$ are the same. On the other hand, the most general linear relationship between $\mathcal{F}_{(\alpha)(\beta)}(\tau)$ and $F_{(\alpha)(\beta)}(\tau)$ consistent with causality is [3]

$$
\begin{aligned}
\mathcal{F}_{(\alpha)(\beta)}(\tau)= & F_{(\alpha)(\beta)}(\tau)+u\left(\tau-\tau_{0}\right) \int_{\tau_{0}}^{\tau} K_{(\alpha)(\beta)}(\gamma)(\delta) \\
& \times F_{(\gamma)(\delta)}\left(\tau^{\prime}\right) d \tau^{\prime},
\end{aligned}
$$

where $u(x)$ is the unit step function such that $u(x)=1$ for $x$ $>0$ and $u(x)=0$ for $x<0$. The kernel $K$ is expected to be directly related to the acceleration of the observer. In Eq. (4), the measured field involves a weighted average over the past worldline of the observer. This is consistent with the viewpoint developed by Bohr and Rosenfeld [4] that a pointwise field determination is not possible in principle and must indeed be replaced by a certain averaging process. Using the decomposition $F_{\mu \nu} \rightarrow(\mathbf{E}, \mathbf{B})$, it is useful to replace $F_{\mu \nu}$ by a column six-vector $F$ that has $\mathbf{E}$ and $\mathbf{B}$ as its components, respectively. Thus Eq. (3) may be expressed as $\hat{F}=\Lambda F$, where $\Lambda(\tau)$ is a $6 \times 6$ matrix and is a representation of the Lorentz group. In this way, Eq. (4) may be written as

$$
\hat{\mathcal{F}}(\tau)=\hat{F}(\tau)+u\left(\tau-\tau_{0}\right) \int_{\tau_{0}}^{\tau} \hat{K}\left(\tau, \tau^{\prime}\right) \hat{F}\left(\tau^{\prime}\right) d \tau^{\prime} .
$$

The Volterra integral equation (5) implies, via Volterra's theorem [5], that in the space of continuous functions the relationship between $\hat{\mathcal{F}}$ and $F$ is unique. Volterra's theorem has been extended to the Hilbert space of square-integrable functions by Tricomi [6]. The ansatz (4) is manifestly Lorentz covariant; moreover, the kernel is given by quantities that are invariant under Poincare transformations of the background Minkowski spacetime.

To determine the kernel $\hat{K}$, we postulate that an electromagnetic radiation field can never stand completely still with respect to an accelerated observer [3]. This is a simple generalization of a well-known result of Lorentz invariance, which may be illustrated using the Doppler effect. If $\omega$ and $\mathbf{k}$ are the frequency and wave vector of an electromagnetic wave with respect to static inertial observers, then an inertial observer moving with velocity $\mathbf{v}$ would measure a frequency $\omega^{\prime}=\gamma(\omega-\mathbf{v} \cdot \mathbf{k})$. This implies that $\omega^{\prime}=0$ if and only if $\omega=0$.
Imposing the same requirement in the general noninertial case, we conclude that if $\hat{\mathcal{F}}$ turns out to be constant in time, then $F$ must have been a constant field in the first place. This requirement leads to an integral equation that could be solved in principle to determine $\hat{K}[3]$. The Volterra-Tricomi uniqueness theorem then implies that for any realistic radiation field $F$, the measured field $\hat{\mathcal{F}}$ will definitely depend upon time. A detailed analysis [7-9] reveals that the unique kernel of the nonlocal theory of accelerated observers is given by $\hat{K}\left(\tau, \tau^{\prime}\right)=\hat{k}\left(\tau^{\prime}\right)$, where

$$
\hat{k}\left(\tau^{\prime}\right)=-\frac{d \Lambda\left(\tau^{\prime}\right)}{d \tau^{\prime}} \Lambda^{-1}\left(\tau^{\prime}\right) .
$$

Some of the observational consequences of this nonlocal theory of accelerated observers have been worked out in the case of linearly accelerated observers [10]; therefore, the present paper is devoted to the observational consequences of the theory for rotating observers. These are worked out in Sec. II for a uniformly rotating observer. Indirect and qualitative evidence in support of the theory is presented in Secs. III, IV, and V. Section VI contains a discussion of our results. Mathematical details are relegated to the appendices.

\section{ROTATION-INDUCED NONLOCALITY}

We now consider the application of the nonlocal theory of accelerated systems to the important special case of a uniformly rotating observer in the $(x, y)$ plane. Let us assume that for $-\infty<t<0$, the observer moves along the straight line parallel to the $y$ axis at $x=r>0$ with constant speed $v$ and arrives at $x=r$ and $y=0$ at $t=0$. From this instant on, it is forced to move in the positive sense on a circle of radius $r$ with uniform frequency $\Omega=v / r$. The azimuthal angle that indicates the position of the observer for $t \geqslant 0$ is given by $\varphi=\Omega t=\gamma \Omega \tau$, where $\gamma$ is the Lorentz factor corresponding to $\beta=v / c=r \Omega / c$. For $t \geqslant 0$, the natural tetrad frame of the observer is given by

$$
\begin{gathered}
\lambda_{(0)}^{\mu}=\gamma(1,-\beta \sin \varphi, \beta \cos \varphi, 0), \\
\lambda_{(1)}^{\mu}=(0, \cos \varphi, \sin \varphi, 0), \\
\lambda_{(2)}^{\mu}=\gamma(\beta,-\sin \varphi, \cos \varphi, 0), \\
\lambda_{(3)}^{\mu}=(0,0,0,1),
\end{gathered}
$$

with respect to the global inertial coordinates $(t, x, y, z)$. In this case, Eq. (3) implies that

$$
\Lambda=\left[\begin{array}{cc}
\Lambda_{1} & \Lambda_{2} \\
-\Lambda_{2} & \Lambda_{1}
\end{array}\right]
$$

where 


$$
\begin{gathered}
\Lambda_{1}=\left[\begin{array}{ccc}
\gamma \cos \varphi & \gamma \sin \varphi & 0 \\
-\sin \varphi & \cos \varphi & 0 \\
0 & 0 & \gamma
\end{array}\right], \\
\Lambda_{2}=\beta \gamma\left[\begin{array}{ccc}
0 & 0 & 1 \\
0 & 0 & 0 \\
-\cos \varphi & -\sin \varphi & 0
\end{array}\right] .
\end{gathered}
$$

Moreover, it follows from Eq. (1) that the observer has invariant translational centripetal acceleration $\tilde{\mathbf{g}}$ $=-v \gamma^{2} \Omega(1,0,0)$ and rotational frequency $\tilde{\boldsymbol{\Omega}}=\gamma^{2} \Omega(0,0,1)$ that are defined with respect to the spatial axes $\lambda_{(i)}^{\mu}, i$ $=1,2,3$, that correspond to the radial, tangential, and $z$ directions, respectively. In terms of these components of the acceleration tensor, the kernel (6) turns out to be a constant $6 \times 6$ matrix given by

$$
\hat{k}=\left[\begin{array}{cc}
\widetilde{\mathbf{\Omega}} \cdot \mathbf{I} & -\widetilde{\mathbf{g}} \cdot \mathbf{I} \\
\widetilde{\mathbf{g}} \cdot \mathbf{I} & \widetilde{\mathbf{\Omega}} \cdot \mathbf{I}
\end{array}\right],
$$

where $I_{i},\left(I_{i}\right)_{j k}=-\epsilon_{i j k}$, is a $3 \times 3$ matrix proportional to the operator of infinitesimal rotations about the $x^{i}$ axis. It follows that the electromagnetic field as measured by the rotating observer for $t \geqslant 0$ is given by

$$
\begin{aligned}
\mathcal{E}_{1}= & \gamma\left(\cos \varphi E_{1}+\sin \varphi E_{2}\right)+\beta \gamma B_{3}+\gamma^{2} \Omega \int_{0}^{\tau}\left(\sin \varphi^{\prime} E_{1}\right. \\
& \left.-\cos \varphi^{\prime} E_{2}\right) d \tau^{\prime}, \\
\mathcal{E}_{2}= & \sin \varphi E_{1}+\cos \varphi E_{2}+\gamma \Omega \int_{0}^{\tau}\left(\cos \varphi^{\prime} E_{1}+\sin \varphi^{\prime} E_{2}\right) d \tau^{\prime}, \\
\mathcal{E}_{3}= & \gamma E_{3}-\beta \gamma\left(\cos \varphi B_{1}+\sin \varphi B_{2}\right)+\beta \gamma^{2} \Omega \int_{0}^{\tau}\left(-\sin \varphi^{\prime} B_{1}\right. \\
& \left.+\cos \varphi^{\prime} B_{2}\right) d \tau^{\prime}, \\
\mathcal{B}_{1}= & \gamma\left(\cos \varphi B_{1}+\sin \varphi B_{2}\right)-\beta \gamma E_{2}+\gamma^{2} \Omega \int_{0}^{\tau}\left(\sin \varphi^{\prime} B_{1}\right. \\
& \left.-\cos \varphi^{\prime} B_{2}\right) d \tau^{\prime}, \\
\mathcal{B}_{2}=- & \sin \varphi B_{1}+\cos \varphi B_{2}+\gamma \Omega \int_{0}^{\tau}\left(\cos \varphi^{\prime} B_{1}+\sin \varphi^{\prime} B_{2}\right) d \tau^{\prime}, \\
\mathcal{B}_{3}= & \gamma B_{3}+\beta \gamma\left(\cos \varphi E_{1}+\sin \varphi E_{2}\right)+\beta \gamma^{2} \Omega \int_{0}^{\tau}\left(\sin \varphi^{\prime} E_{1}\right. \\
& \left.-\cos \varphi^{\prime} E_{2}\right) d \tau^{\prime} .
\end{aligned}
$$

Let us next consider a normally incident plane monochromatic wave of frequency $\omega$ given by

$$
F_{ \pm}(t, \mathbf{x})=i \omega a\left[\begin{array}{l}
\mathbf{e}_{ \pm} \\
\mathbf{b}_{ \pm}
\end{array}\right] e^{-i \omega(t-z / c)},
$$

where $a$ is a complex amplitude, $\mathbf{e}_{ \pm}=\mp(\hat{\mathbf{x}} \pm i \hat{\mathbf{y}}) / \sqrt{2}, \quad \mathbf{b}_{ \pm}$ $=\mp i \mathbf{e}_{ \pm}$and the upper (lower) sign represents positive (negative) helicity radiation. Here, $\mathbf{e}_{ \pm}$and $\mathbf{b}_{ \pm}$are unit circular polarization vectors such that $\mathbf{e}_{ \pm}^{*}=-\mathbf{e}_{\mp}$ and $\mathbf{e}_{ \pm} \cdot \mathbf{e}_{ \pm}^{*}=1$. As usual, we define the intensity of the radiation field (12) to be $I_{0}$ $=(1 / 2) \omega^{2}|a|^{2}$. In employing complex fields, such as in Eq. (12), we adopt the convention that only the real part of the field is of physical interest. This is compatible with our general linear approach based on the superposition principle.

Along the worldline of the rotating observer $z=0$ and $t$ $=\gamma \tau$ in Eq. (12), so that from $\hat{F}=\Lambda F$ we obtain in accordance with the hypothesis of locality

$$
\hat{F}_{ \pm}(\tau)=i \gamma \omega a\left[\begin{array}{c}
\hat{\mathbf{e}}_{ \pm} \\
\hat{\mathbf{b}}_{ \pm}
\end{array}\right] e^{-i \hat{\omega} \tau},
$$

where $\hat{\mathbf{b}}_{ \pm}=\mp i \hat{\mathbf{e}}_{ \pm}$and

$$
\hat{\mathbf{e}}_{ \pm}=\mp \frac{1}{\sqrt{2}}\left[\begin{array}{c}
1 \\
\pm i \gamma^{-1} \\
\pm i \beta
\end{array}\right]
$$

are unit vectors such that $\hat{\mathbf{e}}_{ \pm} \cdot \hat{\mathbf{e}}_{ \pm}^{*}=1$ and $\hat{\mathbf{e}}_{ \pm} \rightarrow \mathbf{e}_{ \pm}$as $\beta \rightarrow 0$. Moreover, $\hat{\omega}=\gamma(\omega \mp \Omega)$ in Eq. (13), so that the transverse Doppler effect is modified by the helicity-rotation coupling. The observational evidence in support of the helicity-rotation coupling for $\omega \gg \Omega$ is discussed in Refs. [11-13]. The general spin-rotation-gravity coupling has been reviewed in Ref. [14].

It follows from Eq. (11) that the nonlocal field measured by the rotating observer is given by (cf. Appendix A)

$$
\hat{\mathcal{F}}_{ \pm}=i \gamma \omega a\left[\begin{array}{c}
\hat{\mathbf{e}}_{ \pm} \\
\hat{\mathbf{b}}_{ \pm}
\end{array}\right] \frac{e^{-i \hat{\omega} \tau} \mp \frac{\Omega}{\omega}}{1 \mp \frac{\Omega}{\omega}} .
$$

It is important to recognize two aspects of this nonlocal result as compared to the local result given by Eq. (13): (i) The oscillatory part of Eq. (15) is the same as in Eq. (13) except for the multiplicative factor of $(1 \mp \Omega / \omega)^{-1}$. It follows from this feature that the measured intensity of the positive helicity incident wave with $\omega>\Omega$ is enhanced by a factor of (1 $-\Omega / \omega)^{-2}$, while the corresponding negative helicity intensity is diminished by a factor of $(1+\Omega / \omega)^{-2}$. Thus for the same incident frequency $\omega$ and intensity $I_{0}$, the ratio of the measured intensity of the positive helicity radiation $I_{+}$to the measured intensity of the negative helicity radiation $I_{-}$is

$$
\frac{I_{+}}{I_{-}}=\left(\frac{\omega+\Omega}{\omega-\Omega}\right)^{2} .
$$

(ii) In contrast with Eqs. (12) and (13), Eq. (15) contains a constant part proportional to $\Omega$; in fact, this constant term is necessary in order that $\hat{\mathcal{F}}_{+}(\tau)$ would have a proper limit in the resonance case $\omega=\Omega$. Indeed, for $\omega=\Omega$ we have $\hat{\omega}=0$ in 
the positive helicity case, $\hat{\omega}=2 \gamma \Omega$ in the negative helicity case and

$$
\hat{\mathcal{F}}_{ \pm}(\tau)=i \gamma \Omega a\left[\begin{array}{c}
\hat{\mathbf{e}}_{ \pm} \\
\hat{\mathbf{b}}_{ \pm}
\end{array}\right] f_{ \pm}(\tau),
$$

where $f_{+}=1-i \gamma \Omega \tau$ and $f_{-}=\cos (\gamma \Omega \tau) \exp (-i \gamma \Omega \tau)$. Thus in the positive helicity case the measured field grows indefinitely with proper time. This linear divergence of the field is a consequence of the fact that the incident plane wave has a constant amplitude for all time; that is, the divergence would disappear for any finite incident wave packet. Let us note that in the corresponding negative helicity case, the measured intensity is given by $I_{-}=(1 / 2) \gamma^{2} I_{0}$. It follows that for $\omega=\Omega, I_{+} / I_{-} \rightarrow \infty$ as $\Omega \tau \rightarrow \infty$.

These predictions of the nonlocal theory of accelerated observers follow directly from the nonlocality of our ansatz (4). It is therefore important to provide observational evidence for these predictions.

Available data regarding rotating systems involve situations with $\omega \gg \Omega$ and appear to be consistent with $I_{+} \approx I_{-}$and the nonlocal theory within the limits of the accuracy of the observations [13]. Imagine, for instance, radio waves of frequency $10 \mathrm{GHz}$ normally incident on a disk that is rotating very rapidly at a rate of $10^{3}$ rounds per second; then, $\Omega / \omega$ $=10^{-7}$ and $I_{+} / I_{-} \approx 1+4 \Omega / \omega$ in this case. The nonrelativistic nature of this thought experiment should be noted: If the disk has a radius of $5 \mathrm{~cm}$, then $v / c \approx 10^{-6}$ at the rim of the disk. It is important to emphasize that the nonlocal theory involves the properties of pure vacuum, whereas in an actual experiment one works with rotating devices whose characteristics must therefore be known to high accuracy. An interesting discussion of this point in the case of the emission of radiation by a rotating atomic system is contained in Ref. [15]. Nevertheless, one can hope that future experiments may achieve levels of accuracy that would make it possible to test predictions (i) and (ii). In the absence of relevant experimental data of high accuracy, however, it is useful to employ Bohr's correspondence principle and determine whether quantum mechanical results are in qualitative agreement with the predictions of the classical nonlocal theory. This is the subject of the sections that follow.

Specifically, in Secs. III and IV we imagine the rotating observer to be an electron in a circular Rydberg state of the hydrogen atom. We then consider a circularly polarized photon that is incident along the normal to the orbital plane. To study the helicity dependence of the intensity of the radiation field experienced by the electron, we calculate the dependence of the ionization cross section of the electron upon the helicity of the incident radiation. To this end, we employ nonrelativistic quantum mechanics and ignore the spin of the electron. In Sec. V, we imagine the rotating observer in (ii) to be an electron in a circular "orbit" around a uniform magnetic field. Classically, the circular electron orbit has rotation frequency $\Omega_{c}$, which is the cyclotron frequency. We study the helicity dependence of the dipole transition rates involving a normally incident photon of frequency $\omega=\Omega_{c}$.

The main objective of these calculations is to learn via the correspondence principle what quantum mechanics can teach us about the physics of accelerated systems [16]. The results of these studies, based on nonrelativistic quantum mechanics, qualitatively bear out, in the correspondence limit, the consequences of the nonlocal theory of uniformly rotating observers.

It is important to note that, due to the nature of the subject matter, the notation employed in the following sections is independent of Secs. I and II, except when otherwise indicated.

\section{HYPOTHESIS OF LOCALITY IN QUANTUM MECHANICS}

The hypothesis of locality is necessary for the extension of quantum mechanics to noninertial frames of reference [17]. Moreover, the impulse approximation scheme in quantum scattering theory, first discussed by Fermi $[18,19]$, turns out to be an application of the hypothesis of locality to quantum particles. To illustrate this point, the impulse approximation is employed in this section to show that the cross section for the ionization of the circular states of atomic hydrogen by a normally incident circularly polarized plane wave is independent of the helicity of the radiation in complete correspondence with the standard classical theory (cf. Sec. II).

The hypothesis of locality involves the replacement of an accelerated system, e.g., a bound electron, by a free system that is otherwise the same. For instance, in the ionization of the bound electron by an incident photon, this (impulse) approximation is valid if the photon energy is much larger than the binding energy of the electron. That is, during the interaction, the momentum of the electron does not change appreciably because of its binding force; therefore, the electron may be treated as a free particle. A free particle of momentum $\mathbf{p}=\hbar \mathbf{q}$ has a wave function proportional to $\exp (i \mathbf{q} \cdot \mathbf{x})$; therefore, we must express the normalized wave function $\Psi(\mathbf{x}, t)=\psi(\mathbf{x}) \exp (-i E t / \hbar)$ of the bound electron in terms of the Fourier integral

$$
\psi(\mathbf{x})=\frac{1}{(2 \pi)^{3 / 2}} \int d^{3} q \hat{\psi}(\mathbf{q}) e^{i \mathbf{q} \cdot \mathbf{x}},
$$

where $\hat{\psi}(\mathbf{q})$ is in effect the momentum-space wave function given by

$$
\hat{\psi}(\mathbf{q})=\frac{1}{(2 \pi)^{3 / 2}} \int d^{3} x \psi(\mathbf{x}) e^{-i \mathbf{q} \cdot \mathbf{x}} .
$$

To illustrate this application of the hypothesis of locality, we consider the circular states of the electron in the hydrogen atom and assume that ionization occurs due to the absorption of a perpendicularly incident photon of frequency $\omega$ such that $M c^{2} \gg \hbar \omega \gg\left|E_{n}\right|$, where $M$ is the mass of the electron and $\left|E_{n}\right|$ is the electron binding energy. The electron in the final state is free, i.e., we neglect the Coulomb interaction. The impulse approximation is valid so long as during the interaction the net impulse due to the Coulomb force can be neglected.

The bound states of the electron in the hydrogen atom are given by the normalized wave functions 


$$
\Psi_{n \ell m}(r, \vartheta, \varphi, t)=R_{n \ell}(r) Y_{\ell m}(\vartheta, \varphi) e^{-\frac{i E_{n} t}{\hbar}}
$$

The circular states with $n>1, \ell=n-1$ and $m= \pm \ell$ correspond to classical circular orbits in the $(x, y)$ plane. The normalized radial part of the wave function for $\ell=n-1$ is

$$
R_{n n-1}(r)=\left(\frac{2}{a_{0} n}\right)^{3 / 2} \frac{1}{\sqrt{(2 n) !}}\left(\frac{2 r}{a_{0} n}\right)^{n-1} e^{-\frac{r}{a_{0} n}},
$$

where $a_{0}=\hbar^{2} /\left(M e^{2}\right)$ is the Bohr radius, $E_{n}=-e^{2} /\left(2 r_{n}\right), r_{n}$ $=a_{0} n^{2}$, and $-e$ is the charge of the electron. We assume for the sake of simplicity that the proton is in effect fixed at the origin of our spherical polar coordinate system. It follows from Eq. (21) that $\langle r\rangle_{n}=n(n+1 / 2) a_{0}$, so that for $n \gg 1$, $\langle r\rangle_{n} \rightarrow r_{n}$.

The circular states of atomic hydrogen have been the subject of extensive experimental studies, especially in the case of Rydberg atoms (see Refs. [20,21], and references therein). In connection with the ionization of atoms in the correspondence regime, it is interesting to note that experimental and theoretical studies have been carried out regarding the ionization of Rydberg atoms by circularly polarized microwave radiation (see Refs. [22], [23], and references therein).

Let us now assume an initial counterclockwise circular state with $m=n-1$ and consider an incident electromagnetic radiation field given by the vector potential $\mathcal{A}$ such that $\boldsymbol{\nabla} \cdot \mathcal{A}=0$. In this Coulomb gauge, $\mathbf{p}$ and $\mathcal{A}$ commute and the interaction Hamiltonian can be written as

$$
\mathcal{H}_{\text {int }}=\frac{e}{M c} \mathcal{A} \cdot \mathbf{p}+\frac{e^{2} \mathcal{A}^{2}}{2 M c^{2}} .
$$

The vector potential may be expressed as

$$
\mathcal{A}=\sum_{\mathbf{k} \epsilon} \sqrt{\frac{2 \pi \hbar c^{2}}{\omega V}}\left(\mathbf{e}_{\mathbf{k} \epsilon} a_{\mathbf{k} \epsilon} e^{-i \omega t+i \mathbf{k} \cdot \mathbf{x}}+\mathbf{e}_{\mathbf{k} \epsilon}^{*} a_{\mathbf{k} \epsilon}^{\dagger} e^{i \omega t-i \mathbf{k} \cdot \mathbf{x}}\right),
$$

where $V$ is the volume of space within a large cube, $\epsilon$ is either plus or minus, and $\omega=c k$. Here, the circular polarization basis for a photon of wave vector $\mathbf{k}$ is denoted by $\mathbf{e}_{\mathbf{k} \epsilon}$; these are so defined that for a photon propagating along the positive $z$ direction $\mathbf{e}_{\mathbf{k} \pm} \rightarrow \mathbf{e}_{ \pm}$given in Sec. II.

We are interested in the ionization cross section due to the absorption of a photon incident along the $z$ axis. The initial $(|i\rangle)$ and final $(|f\rangle)$ states are unperturbed energy eigenstates; indeed, each is a product of the electron state and the photon state. It follows from the standard first-order time-dependent perturbation theory that the rate of transition from an initial state to a final state in which the electron is free is given by

$$
d W=\frac{2 \pi}{\hbar}\left|\mathcal{H}_{f i}\right|^{2} \delta\left(E_{f}-E_{i}\right) \rho_{f} d E_{f}
$$

where $\mathcal{H}_{f i}=\left\langle f\left|\mathcal{H}_{\text {int }}\right| i\right\rangle, \rho_{f}$ is the density of final states, i.e., the number of final states per unit energy. The flux of the incident photon is $c / V$; therefore, the differential cross section for this process is $d \sigma=d W /(c / V)$. Using energy conservation

$$
E_{n}+\hbar \omega=\frac{\hbar^{2} k^{\prime 2}}{2 M}
$$

where $E_{i}=E_{n}+\hbar \omega$ and $E_{f}=\hbar^{2} k^{\prime 2} /(2 M)$, and the expression for $\rho_{f}$

$$
\rho_{f}=\frac{V}{(2 \pi)^{3}} \frac{M k^{\prime}}{\hbar^{2}} d \Omega^{\prime},
$$

where $d k_{x}^{\prime} d k_{y}^{\prime} d k_{z}^{\prime}=k^{\prime 2} d k^{\prime} d \Omega^{\prime}$ and $\Omega^{\prime}$ is the solid angle associated with the final momentum of the electron $\left(\hbar \mathbf{k}^{\prime}\right)$, we find

$$
\frac{d \sigma}{d \Omega^{\prime}}=\frac{M k^{\prime} V^{2}}{4 \pi^{2} c \hbar^{3}}\left|H_{f i}\right|^{2}
$$

Here, $H_{f i}$ is the reduced matrix element connecting only the electronic states for which the interaction Hamiltonian reduces to

$$
H_{\mathrm{int}}=\frac{e}{M} \sqrt{\frac{2 \pi \hbar}{\omega V}} e^{i \mathbf{k} \cdot \mathbf{x}} \mathbf{e}_{ \pm} \cdot \mathbf{p}
$$

where we have assumed that $e|\boldsymbol{A}| / c$ is much smaller than the electron momentum, so that the term proportional to $\mathcal{A}^{2}$ in Eq. (22) may be neglected. Thus

$$
H_{f i}=\int d^{3} x \psi_{f}^{*} H_{\mathrm{int}} \psi_{i}
$$

where $\psi_{f}=V^{-1 / 2} \exp \left(i \mathbf{k}^{\prime} \cdot \mathbf{x}\right)$ and

$$
\psi_{i}=\frac{1}{(2 \pi)^{3 / 2}} \int d^{3} q \hat{\psi}_{n}(\mathbf{q}) e^{i \mathbf{q} \cdot \mathbf{x}} .
$$

We find that

$$
H_{f i}=\frac{4 \pi^{2} e \hbar^{3 / 2}}{M V \omega^{1 / 2}} \mathbf{e}_{ \pm} \cdot\left(\mathbf{k}^{\prime}-\mathbf{k}\right) \hat{\psi}_{n}\left(\mathbf{k}^{\prime}-\mathbf{k}\right),
$$

where $\mathbf{e}_{ \pm} \cdot \mathbf{k}=0$ by assumption and $\mathbf{e}_{ \pm} \cdot \mathbf{k}^{\prime}=\mp\left(k_{x}^{\prime} \pm i k_{y}^{\prime}\right) / \sqrt{2}$. Thus

$$
\frac{d \sigma}{d \Omega^{\prime}}=\frac{2 \pi^{2} \alpha \hbar k^{\prime}}{M \omega}\left(k_{x}^{\prime 2}+k_{y}^{\prime 2}\right)\left|\hat{\psi}_{n}\left(\mathbf{k}^{\prime}-\mathbf{k}\right)\right|^{2},
$$

where $\alpha=e^{2} /(\hbar c)$ is the fine-structure constant. It is already clear from Eq. (32) that there is no helicity-dependent photoionization in the impulse approximation. The general nature of this result should be emphasized, since it is independent of the nature of the initial state.

For comparison purposes, it is useful to compute $d \sigma / d \Omega^{\prime}$ explicitly. To this end, we note that (see Appendix B)

$$
\hat{\psi}_{n}(\mathbf{q})=\frac{1}{2 \pi} \sqrt{\frac{2}{a_{0}}}\left(2 a_{0} n\right)^{n+1} \frac{\left(i q e^{\left.i \phi_{q} \sin \theta_{q}\right)^{n-1}}\right.}{\left(1+a_{0}^{2} n^{2} q^{2}\right)^{n+1}},
$$

where $\hat{\mathbf{q}}=\left(\theta_{q}, \phi_{q}\right)$. From $\hat{\mathbf{k}}^{\prime}=(\theta, \phi)$ and $\mathbf{q}=\mathbf{k}^{\prime}-\mathbf{k}$, we find that

$$
q \sin \theta_{q}=k^{\prime} \sin \theta, \quad q \cos \theta_{q}=k^{\prime} \cos \theta-k, \quad \phi_{q}=\phi .
$$

Thus Eq. (32) may be written as 


$$
\frac{d \sigma}{d \Omega^{\prime}}=4 \alpha a_{0}^{2} n^{3} \frac{\left|E_{n}\right|}{\hbar \omega}\left(2 a_{0} n k^{\prime}\right)^{2 n+1} \frac{\sin ^{2 n} \theta}{\left(1+a_{0}^{2} n^{2} q^{2}\right)^{2 n+2}},
$$

where $q^{2}=k^{2}+k^{\prime 2}-2 k k^{\prime} \cos \theta$. In the impulse approximation, $M c^{2} \gg \hbar \omega \gg\left|E_{n}\right|$; therefore, $k / k^{\prime} \approx v^{\prime} /(2 c) \ll 1$,

$$
a_{0} n k^{\prime} \approx\left(\frac{\hbar \omega}{\left|E_{n}\right|}\right)^{\frac{1}{2}}, \quad 1+a_{0}^{2} n^{2} q^{2} \approx \frac{\hbar \omega}{\left|E_{n}\right|}\left(1-\frac{v^{\prime}}{c} \cos \theta\right),
$$

where $v^{\prime}=\hbar k^{\prime} / M$. Hence, we find that

$$
\frac{d \sigma}{d \Omega^{\prime}} \approx 2^{2 n+3} \alpha a_{0}^{2} n^{3}\left(\frac{\left|E_{n}\right|}{\hbar \omega}\right)^{n+\frac{5}{2}} \frac{\sin ^{2 n} \theta}{\left(1-\frac{v^{\prime}}{c} \cos \theta\right)^{2 n+2}}
$$

The total ionization cross section is then (see Appendix B)

$$
\sigma \approx 2^{4 n+5} \pi \alpha a_{0}^{2} n^{3} \frac{(n !)^{2}}{(2 n+1) !}\left(\frac{\left|E_{n}\right|}{\hbar \omega}\right)^{n+\frac{5}{2}},
$$

which is valid to $O\left(v^{\prime 2} / c^{2}\right)$, since the term linear in $v^{\prime} / c$ vanishes. Though this formula has been derived for circular states $(n>1)$, it applies equally well to the $n=1$ spherically symmetric ground state of the hydrogen atom [24].

The calculation of $\sigma$ has been based on the impulse approximation, which means that during the electron-photon interaction, the change in the momentum of the electron due to the Coulomb binding force has been neglected. However, the ionization cross section is expected to become dependent upon the helicity of the incident radiation when the Coulomb interaction is fully taken into account.

\section{HELICITY-DEPENDENT PHOTOEFFECT}

The standard treatment of the photoeffect is contained in Refs. [25,26]. The purpose of this section is to go beyond the impulse approximation of Sec. III and show that the inclusion of the Coulomb interaction in the final state leads to the helicity-dependent photoeffect that is in qualitative agreement with the nonlocal prediction (i) of Sec. II.

As in Sec. III, we assume that a hydrogen atom is initially in a circular state of energy $E_{n}$. A photon with wave vector $\mathbf{k}=(\omega / c) \hat{\mathbf{z}}$ is normally incident on the electron orbit such that

$$
M c^{2} \gg \hbar \omega>\left|E_{n}\right|,
$$

leading to ionization. In dealing with the reduced matrix element $H_{f i}$, we take the Coulomb interaction into account in the final state wave function. But to simplify matters, we use the electric dipole approximation, i.e., $\exp (i \mathbf{k} \cdot \mathbf{x}) \approx 1$. It follows that $\omega r_{n} \ll c$, where $r_{n}=a_{0} n^{2}$, and hence,

$$
\frac{1}{2} \alpha \ll \frac{\left|E_{n}\right|}{\hbar \omega}<1
$$

Let $\psi_{n}$ be the initial state and $\psi_{C}$ be the final Coulomb state, then

$$
H_{f i}^{ \pm}=\frac{e}{M} \sqrt{\frac{2 \pi \hbar}{\omega V}} \int d^{3} x \psi_{C}^{*}\left(\mathbf{e}_{ \pm} \cdot \mathbf{p}\right) \psi_{n} .
$$

We can replace the momentum operator $\mathbf{p}$ with $(-i M / \hbar)$ $\times\left[\mathbf{x}, H_{0}\right]$, where $H_{0}$ is the unperturbed hydrogen Hamiltonian. It follows from energy conservation, Eq. (25), that

$$
H_{f i}^{ \pm}=i e \sqrt{\frac{2 \pi \hbar \omega}{V}} \int d^{3} x \psi_{C}^{*}\left(\mathbf{e}_{ \pm} \cdot \mathbf{x}\right) \psi_{n} .
$$

We note that

$$
\mathbf{e}_{ \pm} \cdot \mathbf{x}=\sqrt{\frac{4 \pi}{3}} r Y_{1 \pm 1}(\vartheta, \varphi)
$$

Moreover, $\psi_{C}^{*}$ can be expressed as (see Ref. [19], p. 470)

$$
\psi_{C}^{*}=\frac{4 \pi}{\sqrt{V}} \sum_{\ell m} i^{-\ell} C_{\ell}\left(k^{\prime} ; r\right) Y_{\ell m}(\theta, \phi) Y_{\ell m}^{*}(\vartheta, \varphi),
$$

where

$$
\begin{aligned}
C_{\ell}\left(k^{\prime} ; r\right)= & \frac{\left(2 k^{\prime} r\right)^{\ell} e^{\frac{1}{2} \pi \gamma+i k^{\prime} r}}{(2 \ell+1) !} \Gamma(\ell+1-i \gamma) \\
& \times F\left(\ell+1-i \gamma, 2 \ell+2,-2 i k^{\prime} r\right) .
\end{aligned}
$$

Here, $\gamma^{-1}=k^{\prime} a_{0}$ and $F$ is a confluent hypergeometric function. Let us note that if $C_{\ell}\left(k^{\prime} ; r\right)$ is replaced by $j_{\ell}\left(k^{\prime} r\right)$ in Eq. (44), then $\psi_{C}^{*} \rightarrow \psi_{f}^{*}$ of Sec. III. Equation (42) can be expressed as

$$
H_{f i}^{ \pm}=\frac{8 \pi^{2} i e}{V} \sqrt{\frac{2 \hbar \omega}{3}} \sum_{\ell m} i^{-\ell} I_{\ell m}^{ \pm} \mathcal{C}_{\ell} Y_{\ell m}(\theta, \phi),
$$

where

$$
\begin{gathered}
I_{\ell m}^{ \pm}=\int d \Omega Y_{\ell m}^{*}(\Omega) Y_{1 \pm 1}(\Omega) Y_{n-1 n-1}(\Omega), \\
\mathcal{C}_{\ell}=\int_{0}^{\infty} d r r^{3} R_{n n-1}(r) C_{\ell}\left(k^{\prime} ; r\right) .
\end{gathered}
$$

It follows from a standard result (see Ref. [19], p. 290) that

$$
I_{\ell m}^{ \pm}=\sqrt{\frac{3(2 n-1)}{4 \pi(2 \ell+1)}}\left\langle\ell^{\prime}, \ell^{\prime}, 1, \pm 1 \mid \ell, m\right\rangle\left\langle\ell^{\prime}, 0,1,0 \mid \ell, 0\right\rangle,
$$

where $\ell^{\prime}=n-1$. From the general properties of the ClebschGordan coefficients, it is clear that $I_{\ell m}^{+}$can be nonzero only for $\ell=m=n$, while $I_{\ell m}^{-}$can be nonzero only for $m=n-2$ and $\ell=n, n-1, n-2$. Using the table on p. 220 and formula (34.40) on p. 290 of Ref. [19], one finds that

$$
\begin{gathered}
I_{n n}^{+}=\sqrt{\frac{3 n}{4 \pi(2 n+1)}}, \quad I_{n n-2}=\sqrt{\frac{3}{4 \pi\left(4 n^{2}-1\right)},} \\
I_{n-1 n-2}^{-}=0, \quad I_{n-2 n-2}^{-}=-\sqrt{\frac{3(n-1)}{4 \pi(2 n-1)}} .
\end{gathered}
$$

Therefore, 


$$
\begin{gathered}
H_{f i}^{+}=-\frac{4 \pi e}{V i^{n+1}} \sqrt{\frac{2 \pi n \hbar \omega}{2 n+1}} \mathcal{C}_{n} Y_{n n}(\theta, \phi), \\
H_{f i}^{-}=-\frac{4 \pi e}{V i^{n+1}} \sqrt{\frac{2 \pi(n-1) \hbar \omega}{2 n-1}}\left[\mathcal{C}_{n-2} Y_{n-2}{ }_{n-2}(\theta, \phi)\right. \\
\left.+\frac{1}{\sqrt{(n-1)(2 n+1)}} \mathcal{C}_{n} Y_{n n-2}(\theta, \phi)\right] .
\end{gathered}
$$

Computing the total cross section

$$
\sigma_{ \pm}=\frac{M k^{\prime} V^{2}}{4 \pi^{2} c \hbar^{3}} \int\left|H_{f i}^{ \pm}\right|^{2} d \Omega^{\prime},
$$

we find that

$$
\begin{gathered}
\sigma_{+}=8 \pi \frac{k k^{\prime}}{a_{0}} \frac{n}{2 n+1}\left|\mathcal{C}_{n}\right|^{2}, \\
\sigma_{-}=8 \pi \frac{k k^{\prime}}{a_{0}}\left[\frac{n-1}{2 n-1}\left|\mathcal{C}_{n-2}\right|^{2}+\frac{1}{4 n^{2}-1}\left|\mathcal{C}_{n}\right|^{2}\right] .
\end{gathered}
$$

The quantities $\mathcal{C}_{n}$ and $\mathcal{C}_{n-2}$ can be calculated using the results given in Appendix B. Hence,

$$
\begin{aligned}
\mathcal{C}_{n}= & \sqrt{8}\left(a_{0} n\right)^{5 / 2}\left(\frac{4 n}{\gamma}\right)^{n} \frac{\Gamma(n+1-i \gamma)}{\sqrt{(2 n) !}} \\
& \times\left(1+\frac{n^{2}}{\gamma^{2}}\right)^{-n-2} e^{\frac{1}{2} \pi \gamma-2 \gamma \cot ^{-1}\left(\frac{\gamma}{n}\right)} .
\end{aligned}
$$

Similarly, we find that

$$
\frac{\mathcal{C}_{n-2}}{\mathcal{C}_{n}}=\frac{1}{2 n} \frac{n+i \gamma}{n-1-i \gamma} .
$$

It follows from these results that

$$
\frac{\sigma_{-}}{\sigma_{+}}=\frac{3 n^{2}(n-1)+(3 n+1) \gamma^{2}}{4 n^{3}\left[(n-1)^{2}+\gamma^{2}\right]},
$$

which is valid for $n=1,2,3, \ldots$. The $n=1$ ground state of hydrogen is spherically symmetric; therefore, $\sigma_{+}=\sigma_{-}$in agreement with Eq. (59). For circular states with $n>1$, Eq. (59) implies that $\sigma_{-}<\sigma_{+}$in correspondence with the nonlocal theory of Sec. II.

To bring out this qualitative agreement more explicitly, we recall that for a Bohr orbit of speed $v_{n}=c \alpha / n$ and radius $r_{n}$, one can define a Bohr frequency $\Omega_{n}$ given by $v_{n}=r_{n} \Omega_{n}$; it is then simple to show that $\Omega_{n}=2\left|E_{n}\right| /(\hbar n)$. Let us consider the ratio $\eta:=\Omega_{n} / \omega$ for a circular state. Then, from Eq. (25) and $\gamma^{-1}=k^{\prime} a_{0}$ we find that

$$
\gamma^{2}=\frac{n^{3} \eta}{2-n \eta}
$$

Substituting this relation in the expression for $\sigma_{-} / \sigma_{+}$results in

$$
\frac{\sigma_{-}}{\sigma_{+}}=\frac{3(n-1)+2 n \eta}{2 n\left[2(n-1)^{2}+n(2 n-1) \eta\right]},
$$

so that for a given circular state $n, \sigma_{-} / \sigma_{+}$only depends on $\eta=\Omega_{n} / \omega$ in agreement with the classical nonlocal theory. We note that Eq. (40) can be written in terms of $\eta$ as

$$
\frac{\alpha}{n} \ll \eta<\frac{2}{n}
$$

therefore, for $n \geqslant 2$ this approach can be qualitatively compared with the nonlocal theory, cf. Eq. (16).

The treatment of ionization presented here can be used near threshold $(\gamma \rightarrow \infty)$ as well as for $\hbar \omega$ in the intermediate energy regime given by $M c^{2} \gg \hbar \omega \gg\left|E_{n}\right|$, where $\gamma \ll 1$. The threshold behavior has been discussed, for instance, in Refs. $[19,26]$; therefore, we concentrate on the latter case $(\gamma \ll 1)$ that was treated in the previous section using the impulse approximation. In general, it is possible to express $n / \gamma$ as

$$
\frac{n}{\gamma}=\sqrt{\frac{\hbar \omega}{\left|E_{n}\right|}-1},
$$

so that for $\gamma \ll 1$, $\hbar \omega \gg\left|E_{n}\right|$ and

$$
\frac{n}{\gamma} \approx\left(\frac{\hbar \omega}{\left|E_{n}\right|}\right)^{1 / 2} \gg 1 \text {. }
$$

It follows that $d \sigma_{+} / d \Omega^{\prime}$ reduces in this case to the result of the impulse approximation $d \sigma / d \Omega^{\prime}$ given in Eq. (37), i.e., for $\gamma \ll 1$;

$$
\frac{d \sigma_{+}}{d \Omega^{\prime}} \approx \frac{d \sigma}{d \Omega^{\prime}} .
$$

For $n \geqslant 2$, however, $d \sigma_{-} / d \Omega^{\prime}$ does not approach $d \sigma / d \Omega^{\prime}$ for $\gamma \ll 1$; in fact, $\sigma_{-} / \sigma_{+} \approx 3 /[4 n(n-1)]$ in this case. This helicity dependence is a noteworthy aspect of the Coulomb interaction. It would be interesting to investigate experimentally this helicity dependence of the photoeffect for $n \geqslant 2$.

\section{RESONANT ABSORPTION}

Let us now consider the motion of an electron in a uniform magnetic field $\mathbf{B}=B \hat{\mathbf{z}}$. We are interested in circular orbits about the magnetic lines of force; therefore, we consider the solution of Schrödinger's equation in cylindrical coordinates $(\rho, \varphi, z)$, i.e.,

$$
\frac{1}{2 M}\left(\mathbf{p}+\frac{e}{c} \mathbf{A}\right)^{2} \Psi=i \hbar \frac{\partial \Psi}{\partial t},
$$

where $\mathbf{A}=(1 / 2) B \rho \hat{\boldsymbol{\varphi}}$. It is useful to introduce a magnetic length $\rho_{0}, \rho_{0}^{2}=\hbar c /(e B)$. The nonrelativistic treatment is valid so long as the Compton wavelength of the electron is much smaller than the magnetic radius $\rho_{0}$; this requirement can be satisfied for $B \ll M^{2} c^{3} /(e \hbar)$.

In terms of a dimensionless radial variable $\xi, \quad \xi$ $=\rho^{2} /\left(2 \rho_{0}^{2}\right)$, the solutions of Eq. (66) can be expressed in terms of the confluent hypergeometric functions [27]. The acceptable solutions of the Schrödinger equation (66) are of the form 


$$
\Psi=C_{0} e^{i m \varphi+i \frac{p_{z}}{\hbar} z-i \frac{E}{\hbar} t} \chi(\xi),
$$

where $C_{0}$ is a normalization constant, $m$ is the azimuthal quantum number, and

$$
\chi(\xi)=\xi^{\frac{1}{2}|m|} e^{-\frac{1}{2} \xi} L_{n_{\rho}}^{|m|}(\xi) .
$$

Here, $n_{\rho}=0,1,2, \ldots$, is the radial quantum number and $L_{n_{\rho}}^{\mid m}$ is an associated Laguerre polynomial (see Appendix C). The energy of the electron is given by

$$
E=\frac{p_{z}^{2}}{2 M}+\hbar \Omega_{c}\left(n+\frac{1}{2}\right),
$$

where $\Omega_{c}=e B /(M c)$ is the cyclotron frequency, $\hbar \Omega_{c} \ll M c^{2}$, and

$$
n=n_{\rho}+\frac{m+|m|}{2}
$$

To discuss the correspondence limit, it is convenient to define the following three Hermitian operators:

$$
H^{c}=\frac{p_{z}^{2}}{2 M}+\frac{1}{2} M \Omega_{c}^{2} \rho^{2}, \quad J^{c}=M \Omega_{c} \rho^{2}
$$

and $\ell=M \mathbf{r} \times \mathbf{v}$, where $\mathbf{v}$ is defined by $\mathbf{p}=M \mathbf{v}-e \mathbf{A} / c$. Let us note that $H^{c}$ corresponds to the classical energy of the electron, $J^{c}$ corresponds to its angular momentum about the $z$ axis and $\ell$ is the operator of the classical orbital angular momentum. Thus $\ell=\mathbf{L}+(e / c) \mathbf{r} \times \mathbf{A}$ and $\ell_{z}=L_{z}+e B \rho^{2} /(2 c)$. We find that in the eigenstate given by Eq. $(67),\langle\xi\rangle=2 n_{\rho}$ $+|m|+1$, so that

$$
\begin{gathered}
\left\langle H^{c}\right\rangle=\frac{p_{z}^{2}}{2 M}+\hbar \Omega_{c}\left(2 n_{\rho}+|m|+1\right), \\
\left\langle J^{c}\right\rangle=2 \hbar\left(2 n_{\rho}+|m|+1\right), \\
\left\langle\ell_{z}\right\rangle=2 \hbar\left(n_{\rho}+\frac{m+|m|}{2}+\frac{1}{2}\right) .
\end{gathered}
$$

Clearly, $\quad H^{c}=p_{z}^{2} /(2 M)+(1 / 2) \Omega_{c} J^{c} \quad$ and $\quad E=p_{z}^{2} /(2 M)$ $+(1 / 2) \Omega_{c}\left\langle\ell_{z}\right\rangle$. We expect that in the correspondence limit $E \sim\left\langle H^{c}\right\rangle$ and, hence, $\left\langle J^{c}\right\rangle \sim\left\langle\ell_{z}\right\rangle$, so that the wave function with $m \gg 1$ and $m \gg n_{\rho}$ would correspond to classical orbits based on the comparison between Eqs. (69) and (72).

We are interested in the transition of the electron to a state of higher energy as a result of the resonant absorption of a photon of frequency $\omega=\Omega_{c}$ that is normally incident along the $z$ direction. We therefore assume that the electron is initially in a circular orbit with energy $\mathcal{E}_{i}, p_{z}=0$, and $n_{i}=n_{\rho}$ $+m_{i}$, where $m_{i} \gg 1$ and $m_{i} \gg n_{\rho}$. Conservation of energy and momentum imply that the excited state should have energy $\mathcal{E}_{f}=\mathcal{E}_{i}+\hbar \omega$ and momentum $p_{z}=\hbar \omega / c$ with $\omega=\Omega_{c}$. Thus the initial and final principal quantum numbers are related by $n_{f}-n_{i}=1-\hbar \Omega_{c} /\left(2 M c^{2}\right)$; however, we neglect $\hbar \Omega_{c} /\left(M c^{2}\right)$ $\ll 1$ in our nonrelativistic approximation scheme and set $n_{f}$ equal to $n_{i}+1$.

The interaction Hamiltonian is given by

$$
\mathcal{H}_{\text {int }}=\frac{e}{M c} \mathcal{A} \cdot\left(\mathbf{p}+\frac{e}{c} \mathbf{A}\right)+\frac{e^{2} \mathcal{A}^{2}}{2 M c^{2}},
$$

where $\mathcal{A}$ is given by Eq. (23). Assuming that the incident radiation is sufficiently weak, i.e., $e|\mathcal{A}| / c$ is very small compared to the electron momentum, we neglect the term proportional to $\mathcal{A}^{2}$ in Eq. (75). The transition probability for the ideal case of resonant absorption can be simply worked out using first-order time-dependent perturbation theory [27], and the result is

$$
P=\frac{1}{\hbar^{2}}\left|\left\langle f\left|\mathcal{H}_{\text {int }}\right| i\right\rangle\right|^{2} t^{2}
$$

clearly, the validity of Eq. (76) is limited in time. Here $|i\rangle$ and $|f\rangle$ are unperturbed energy eigenstates; in fact, each is a product of the electron state and the photon state. To simplify matters, line broadening is totally neglected here; in particular, the unperturbed states are assumed to have infinite lifetimes.

Concentrating on the matrix element $\left\langle f\left|\mathcal{H}_{\text {int }}\right| i\right\rangle$, we note that $\mathcal{H}_{\text {int }}$ reduces to

$$
H_{\mathrm{int}}=\frac{e}{M} \sqrt{\frac{2 \pi \hbar}{\Omega_{c} V}} \mathbf{e}_{ \pm} \cdot\left(\mathbf{p}+\frac{e}{c} \mathbf{A}\right)
$$

that acts only on the electronic states. It can thus be expressed as

$$
H_{\mathrm{int}}= \pm i \hbar e \sqrt{\frac{\pi}{2 M V}} e^{ \pm i \varphi} \xi \frac{1}{2}\left(2 \frac{\partial}{\partial \xi} \pm \frac{i}{\xi} \frac{\partial}{\partial \varphi} \mp 1\right) .
$$

It follows from the conservation of angular momentum that we must have $m_{f}=m_{i} \pm 1$, since a photon of helicity \pm 1 carries an angular momentum of $\pm \hbar$ along its direction of motion. Writing $n_{f}=n_{\rho}^{\prime}+m_{f}$ and $n_{i}=n_{\rho}+m_{i}$, we find that $n_{\rho}^{\prime}=n_{\rho}$ in the positive helicity case and $n_{\rho}^{\prime}=n_{\rho}+2$ in the negative helicity case. Thus the computation of the matrix element $\left\langle f\left|\mathcal{H}_{\text {int }}\right| i\right\rangle$ reduces to the evaluation of the integrals

$$
\mathcal{I}_{ \pm}=\int_{0}^{\infty} \chi_{f}^{*} \xi \frac{1}{2}\left(2 \frac{\partial}{\partial \xi} \mp \frac{m_{i}}{\xi} \mp 1\right) \chi_{i} d \xi
$$

since $\rho d \rho d \varphi=\rho_{0}^{2} d \xi d \varphi$. Here, $\chi_{i}$ and $\chi_{f}$ are given by Eq. (68) for the initial and final states. It turns out that (see Appendix C)

$$
\mathcal{I}_{+}=-2 \frac{\left(n_{\rho}+m_{i}+1\right) !}{n_{\rho} !}, \quad \mathcal{I}_{-}=0 .
$$

The correspondence principle connects the square of the amplitude of the classical field measured by the accelerated observer with the probability of transition. In the positivehelicity case, both of these functions increase quadratically with time, while in the negative-helicity case the classical field is periodic in time and averages to zero in agreement with the fact that the transition probability vanishes in quantum mechanics. We conclude that the qualitative results of first-order perturbation theory for resonant absorption are consistent with the nonlocal electrodynamics of uniformly rotating observers. 


\section{DISCUSSION}

The nonlocal theory of accelerated observers is an attempt at the simplest physical theory that has a consistent mathematical structure and goes beyond the standard theory that is based on the hypothesis of locality, namely, the assumption that an observer's acceleration is irrelevant at each instant for measurement purposes, so that the accelerated observer is equivalent to a hypothetical momentarily comoving inertial observer. The nonlocal theory involves an averaging procedure over the past worldline of an accelerated observer; the corresponding weighting function is a kernel that represents the memory of past acceleration. The consequences of this theory have been worked out in the present paper for a uniformly rotating observer. These are compared with the helicity dependence of the rates of ionization of circular states of atomic hydrogen as well as the helicity dependence of the transition probabilities for electrons in circular "orbits" about a uniform magnetic field. The nonlocal results agree better with quantum mechanics in the correspondence limit than the standard relativistic theory of accelerated observers based on the locality hypothesis.

\section{APPENDIX A}

A simple way to derive Eq. (15) is via the following general result: Substituting Eq. (6) for the kernel in Eq. (5), then using $\hat{F}=\Lambda F$ and integration by parts, we obtain for $\tau>\tau_{0}$

$$
\hat{\mathcal{F}}(\tau)=\hat{F}\left(\tau_{0}\right)+\int_{\tau_{0}}^{\tau} \Lambda\left(\tau^{\prime}\right) \frac{d F}{d \tau^{\prime}} d \tau^{\prime} .
$$

Let us now specialize to the case of a monochromatic radiation field such that $F$ varies with proper time as $\exp (-i \gamma \omega \tau)$; then,

$$
\hat{\mathcal{F}}(\tau)=\hat{F}\left(\tau_{0}\right)-i \gamma \omega \int_{\tau_{0}}^{\tau} \hat{F}\left(\tau^{\prime}\right) d \tau^{\prime} .
$$

Suppose that, as in Eq. (13), $\hat{F}(\tau)$ varies with proper time as $\exp (-i \hat{\omega} \tau)$; then, Eq. (A2) implies that

$$
\hat{\mathcal{F}}(\tau)=\hat{F}\left(\tau_{0}\right)\left[1+\gamma \omega \frac{e^{-i \hat{\omega}\left(\tau-\tau_{0}\right)}-1}{\hat{\omega}}\right] .
$$

Substituting Eq. (13) for $\hat{F}$ and setting $\tau_{0}=0$ in Eq. (A3), we recover Eq. (15).

\section{APPENDIX B}

In computing the Fourier integral of Eq. (20), the following relations have been used:

$$
\begin{gathered}
e^{-i \mathbf{q} \cdot \mathbf{x}}=4 \pi \sum_{\ell m}(-i)^{\ell} j_{\ell}(q r) Y_{\ell m}\left(\theta_{q}, \phi_{q}\right) Y_{\ell m}^{*}(\vartheta, \varphi), \\
Y_{\ell \ell}(\hat{\mathbf{q}})=\frac{(-1)^{\ell}}{2^{\ell} \ell !} \sqrt{\frac{(2 \ell+1) !}{4 \pi}} e^{i \ell \phi_{q}} \sin ^{\ell} \theta_{q},
\end{gathered}
$$

$$
\int_{0}^{\infty} \rho^{n+1} e^{-\rho} j_{n-1}(\lambda \rho) d \rho=2^{n} n ! \frac{\lambda^{n-1}}{\left(1+\lambda^{2}\right)^{n+1}} .
$$

This integral follows from the second formula in (6.623) on p. 712 of Ref. [28], namely,

$$
\int_{0}^{\infty} e^{-\alpha x} J_{\nu}(\beta x) x^{\nu+1} d x=\frac{2 \alpha(2 \beta)^{\nu} \Gamma\left(\nu+\frac{3}{2}\right)}{\sqrt{\pi}\left(\alpha^{2}+\beta^{2}\right)^{\nu+\frac{3}{2}}},
$$

where $\operatorname{Re} \nu>-1$ and $\operatorname{Re} \alpha>|\operatorname{Im} \beta|$. Using

$$
j_{\ell}(\rho)=\sqrt{\frac{\pi}{2 \rho}} J_{\ell+\frac{1}{2}}(\rho),
$$

$\alpha=1, \beta=\lambda$, and $\nu=n+1 / 2$, we get Eq. (B3) with $n \rightarrow n+1$.

The evaluation of the total cross section in Eq. (38) is based on the relation

$$
\int_{0}^{\pi} \sin ^{2 n+1} \theta d \theta=2^{2 n+1} \frac{(n !)^{2}}{(2 n+1) !} .
$$

To calculate $\mathcal{C}_{n}$ and $\mathcal{C}_{n-2}$ in Eqs. (57) and (58), one can use the relation (see Ref. [27], §f of Mathematical Appendices)

$$
\int_{0}^{\infty} e^{-\zeta z} z^{\nu} F(a, b, K z) d z=(-1)^{N} \Gamma(b) \frac{d^{N}}{d \zeta^{N}}\left[\zeta^{a-b}(\zeta-K)^{-a}\right],
$$

where $\nu+1=b+N, N=1,2,3, \ldots$, and

$$
\operatorname{Re}(b-a)>0, \quad \operatorname{Re}(b+N)>0, \quad \operatorname{Re} \zeta>|\operatorname{Re} K| .
$$

To calculate $\mathcal{C}_{n}$, one can change the integration variable in Eq. (48) to $r /\left(a_{0} n\right)$ and let $N=1, \zeta=1-i n / \gamma, \nu=b=2 n+2$, $a=n+1-i \gamma$ and $K=-2 i n / \gamma$. In Eq. (57), we have used the relation

$$
\left(\frac{\gamma+i n}{\gamma-i n}\right)^{i \gamma}=e^{-2 \gamma \cot ^{-1}\left(\frac{\gamma}{n}\right)}
$$

moreover, $\Gamma(n+1-i \gamma)$ can be computed using $\Gamma(1+z)$ $=z \Gamma(z)$ and

$$
\Gamma(z) \Gamma(-z)=-\frac{\pi}{z \sin \pi z} .
$$

It follows that

$$
|\Gamma(n+1-i \gamma)|^{2}=\frac{\pi \gamma}{\sinh \pi \gamma} \prod_{s=1}^{n}\left(s^{2}+\gamma^{2}\right) .
$$

Furthermore, regarding the phase of $\Gamma(n+1-i \gamma)$, we note that [26]

$$
\frac{\Gamma(n+1-i \gamma)}{\Gamma(n+1+i \gamma)} \approx 1-2 i \gamma \ln \left(n+\frac{1}{2}\right)-2 \gamma^{2} \ln ^{2}\left(n+\frac{1}{2}\right)+\cdots
$$

for $\gamma \ll 1$ and $n \gg 1$. More generally, one can use Stirling's series that is an asymptotic expansion given by 
$\ln \Gamma(1+z)=\frac{1}{2} \ln 2 \pi+\left(z+\frac{1}{2}\right) \ln z-z+\sum_{n=1}^{\infty} \frac{B_{2 n}}{2 n(2 n-1)} z^{1-2 n}$

where the $B_{2 n}$ are Bernoulli numbers.

For the calculation of $\mathcal{C}_{n-2}$, we change the integration variable the same way as before and let $N=3, \zeta=1-i n / \gamma$, $\nu=2 n, a=n-1-i \gamma, b=2 n-2$ and, as before, $K=-2 i n / \gamma$. It follows from a long but straightforward calculation that $\mathcal{C}_{n-2}$ is given by Eq. (58).

\section{APPENDIX C}

The associated Laguerre polynomial $L_{n}{ }^{k}(x)$ is defined by the generating function

$$
\frac{e^{-\frac{x z}{1-z}}}{(1-z)^{k+1}}=\sum_{n=0}^{\infty} L_{n}^{k}(x) z^{n}
$$

for $|z|<1$ and $k=0,1,2, \ldots$

In the calculation of $\mathcal{I}_{ \pm}$in Eq. (79), the following relations were used

$$
\begin{gathered}
\frac{d L_{n+1}{ }^{k}(x)}{d x}=-L_{n}^{k+1}(x), \\
L_{n}^{k+1}=L_{n}^{k}+L_{n-1}{ }^{k}+\cdots+L_{0}^{k},
\end{gathered}
$$

as well as the orthogonality property

$$
\int_{0}^{\infty} e^{-x} x^{k} L_{m}{ }^{k} L_{n}{ }^{k} d x=\frac{(n+k) !}{n !} \delta_{m n} .
$$

From Eq. (C3) one gets the useful relation

$$
L_{n}{ }^{k}=L_{n}{ }^{k+1}-L_{n-1}{ }^{k+1} \text {. }
$$

[1] A. Einstein, The Meaning of Relativity (Princeton University Press, Princeton, 1955).

[2] B. Mashhoon, Phys. Lett. A 143, 176 (1990); B. Mashhoon, Phys. Lett. A 145, 147 (1990); B. Mashhoon, in Relativity in Rotating Frames, edited by G. Rizzi and M. L. Ruggiero (Kluwer Academic, Dordrecht, 2003), pp. 43-55.

[3] B. Mashhoon, Phys. Rev. A 47, 4498 (1993).

[4] N. Bohr and L. Rosenfeld, K. Dan. Vidensk. Selsk. Mat. Fys. Medd. 12, No. 8 (1933); N. Bohr and L. Rosenfeld, translated in Quantum Theory and Measurement, edited by J. A. Wheeler and W. H. Zurek (Princeton University Press, Princeton, 1983); N. Bohr and L. Rosenfeld, Phys. Rev. 78, 794 (1950).

[5] V. Volterra, Theory of Functionals and of Integral and IntegroDifferential Equations (Dover, New York, 1959).

[6] F. G. Tricomi, Integral Equations (Interscience, New York, 1957).

[7] C. Chicone and B. Mashhoon, Ann. Phys. (Leipzig) 11, 309 (2002).

[8] C. Chicone and B. Mashhoon, Phys. Lett. A 298, 229 (2002).

[9] F. W. Hehl and Y. N. Obukhov, Foundations of Classical Electrodynamics (Birkhäuser, Boston, 2003).

[10] B. Mashhoon, Phys. Rev. A 70, 062103 (2004).

[11] B. Mashhoon, Phys. Lett. A 139, 103 (1989); B. Mashhoon, R. Neutze, M. Hannam, and G. E. Stedman, Phys. Lett. A 249, 161 (1998); J. C. Hauck and B. Mashhoon, Ann. Phys. (Leipzig) 12, 275 (2003).

[12] N. Ashby, Living Rev. Relativ. 6, 1 (2003).

[13] B. Mashhoon, Phys. Lett. A 306, 66 (2002); J. D. Anderson and B. Mashhoon, ibid. 315, 199 (2003).

[14] B. Mashhoon, Gen. Relativ. Gravit. 31, 681 (1999); B. Mashhoon, Class. Quantum Grav. 17, 2399 (2000).
[15] I. Bialynicki-Birula and Z. Bialynicka-Birula, Phys. Rev. Lett. 78, 2539 (1997)

[16] I owe this idea to S. Chu.

[17] B. Mashhoon, Phys. Rev. Lett. 61, 2639 (1988).

[18] E. Fermi, Ric. Sci. VII-11, 13 (1936); R. G. Newton, Scattering Theory of Waves and Particles, 2nd ed. (Springer-Verlag, New York, 1982); M. L. Goldberger and K. M. Watson, Collision Theory (Wiley, New York, 1964).

[19] K. Gottfried, Quantum Mechanics (Benjamin, New York, 1966).

[20] R. G. Hulet and D. Kleppner, Phys. Rev. Lett. 51, 1430 (1983).

[21] R. Lutwak, J. Holley, P. P. Chang, S. Paine, D. Kleppner, and T. Ducas, Phys. Rev. A 56, 1443 (1997).

[22] P. Fu, T. J. Scholz, J. M. Hettema, and T. F. Gallagher, Phys. Rev. Lett. 64, 511 (1990).

[23] A. F. Brunello, T. Uzer, and D. Farrelly, Phys. Rev. A 55, 3730 (1997); T. Cheng, J. Liu, S. Chen, and H. Guo, Phys. Lett. A 265, 384 (2000).

[24] In connection with $\sigma$ for $n=1$, there is a misprint in Eq. (58.14) of Ref. [19]: the relevant exponent $(n+5 / 2)$ should be $7 / 2$ instead of unity.

[25] M. Stobbe, Ann. Phys. (Leipzig) 7, 661 (1930); H. Hall, Rev. Mod. Phys. 8, 358 (1936).

[26] H. A. Bethe and E. Salpeter, Quantum Mechanics of One-and Two-Electron Atoms (Plenum, New York, 1977).

[27] L. D. Landau and E. M. Lifshitz, Quantum Mechanics (Pergamon, Oxford, 1965).

[28] I. S. Gradshteyn and I. M. Ryzhik, Table of Integrals, Series and Products (Academic Press, New York, 1980). 